\title{
Profilo clinico ed economico di oxatomide
}

Lorenzo Pradelli*, Mario Eandi*

PROFILO

\begin{abstract}
Oxatomide is an antiallergic drug with a double mechanism of action: it possesses antagonistic activity on the $\mathrm{H}_{1}$ histaminergic receptor and it inhibits the production and release of flogistic and allergic mediators by effector cells, presumably acting as a selective calcium channel blocker on these cellular types.

The efficacy and safety of oxatomide have been evaluated in many studies conducted on patients affected by different clinical conditions, demonstrating remarkable flexibility. It proved to be safe and effective also in extreme ages, i.e. children under 3 months of age and elderly over 80 years. Approved indications include rhinitis, asthma, conjunctivitis, urticaria, atopic and allergic dermatitis and food allergy and intolerance.

In direct comparisons, oxatomide consistently proved superior than placebo and equally or more effective than other consolidated antiallergics. The drug tolerability is good, its main side effects are drowsiness and weight gain, usually transient. Cardiac and hepatic safety data are very reassuring, as is the case for drug interaction potential.

Form an economical point of view, the drug acquisition cost is acceptable, being among the lowest in its class, at equally effective doses.

Overall, the efficacy in several clinical conditions, the good tolerability in most patients and the reasonable purchase cost suggest that oxatomide is characterized by excellent risk-to-benefit and cost-to-benefit ratios in the treatment of allergic pathologies.
\end{abstract}

Farmeconomia e percorsi terapeutici 2003; 4 (4): 217-230

\section{INTRODUZIONE}

Le malattie allergiche sono tra le patologie più diffuse nei paesi occidentali, dove la loro prevalenza e gravità è in costante aumento. Per quanto nella maggioranza dei casi le patologie ad eziologia allergica non costituiscano una minaccia per la sopravvivenza, esse sono tuttavia in grado di peggiorare gravemente la qualità di vita dei pazienti e del loro nucleo familiare, esordendo spesso in età infantile.

La migliorata conoscenza della fisiopatologia della malattia allergica, con il riconoscimento del ruolo fondamentale del processo flogistico nella patogenesi e nella progressione dei fenomeni allergici, ha accresciuto l'interesse per i farmaci antiallergici cosiddetti "dual action", che all'efficacia antistaminica $\mathrm{H}_{1}$ uniscono un' azione inibente sui mediatori cellulari dell'infiammazione.

Scopo del presente lavoro è delineare un profilo farmacologico clinico e farmacoeconomico dell' oxatomide, un farmaco appartenente alla classe degli antiallergici "dual action".

La revisione dei principali lavori scientifici pubblicati sull'oxatomide verrà utilizzata come base per condurre un' analisi dei valori clinici ed economici del suo utilizzo, condotta dal punto di vista di tutti i soggetti coinvolti nella pratica clinica.

\section{Farmacodinamica}

Oxatomide è un farmaco in grado di contrastare la reazione allergica agendo in diverse fasi del suo sviluppo. Il primo meccanismo d'azione studiato consiste nell' antagonismo selettivo dei recettori $\mathrm{H}_{1}$-istaminergici [1], che inibisce le azioni dell'istamina, il principale mediatore preformato. L'attività antistaminica dell'oxatomide non si esplica solo a livello recettoriale: il farmaco, infatti, riduce anche il rilascio dell'istamina da parte di mastociti e basofili dopo stimolo, sia immunologo che non [2]. L'attività antiallergica di oxatomide è più completa di quella di un antistaminico puro, comprendendo anche un'azione antinfiammatoria [3].

La reazione infiammatoria-allergica è sostenuta da una serie di cellule effettrici, che vengono suddivise, in base alla sequenza cronologica con cui intervengono nell'evoca-
* Farmacologia Clinica, Università di Torino 
re la risposta, in primarie (basofili, mastociti ed eosinofili) e secondarie (neutrofili, macrofagi e piastrine). [4-6] Tali cellule, in seguito a stimolazione immunologica, innescano la risposta allergica rilasciando mediatori chimici preformati (come l'istamina) e neoformati (leucotrieni, PAF).

L'oxatomide, mediante un'azione sui flussi di calcio, inibisce sia la degranulazione delle cellule effettrici sia la sintesi de novo dei mediatori lipidici della flogosi allergica [7], con conseguente inibizione sia della risposta immediata, sia di quella tardiva.

L'inibizione della degranulazione non si esercita solamente sulle cellule che presentano il recettore delle $\operatorname{IgE}$ (basofili e mastociti), ma è evidente anche in altre cellule infiammatorie, come i granulociti neutrofili.

In queste cellule, oxatomide inibisce l'afflusso di calcio extracellulare attraverso i canali della membrana cellulare e interferisce con il rilascio di calcio dai depositi intracellulari. L'incremento delle concentrazioni di calcio intracellulare appare responsabile dell'esocitosi dei mediatori preformati e dell'attivazione della fosfolipasi $\mathrm{A}_{2}$, l'enzima che mobilizza l'acido arachidonico dai fosfolipidi di membrana, rendendolo disponibile per la sintesi dei nuovi mediatori lipidici, quali i leucotrieni e il PAF.

Oxatomide dunque possiede un'attività antiallergica complessa, che all'azione antistaminica unisce l'inibizione della flogosi che si innesca nel corso delle reazioni allergiche.

L'azione antinfiammatoria è di grande importanza, in quanto è stato documentato che $\mathrm{i}$ soggetti allergici presentano uno stato di flogosi minima persistente anche quando l'esposizione agli allergeni è di intensità insufficiente a provocare manifestazioni sintomatiche. Questa infiammazione costante svolge un importante ruolo nell' evoluzione della patologia allergica, che può passare da una fase in cui le crisi si alternano a periodi di ritorno alla norma a malattia cronica, senza più possibilità di restitutio ad integrum per il carattere permanente del danno tissutale. Inibendo la reazione infiammatoria allergica nelle sue fasi iniziali, oxatomide limita tale rischio.

\section{Farmacocinetica}

Dopo somministrazione orale di una compressa di $60 \mathrm{mg}$ di oxatomide a volontari sani, il farmaco è ben assorbito, raggiungendo il picco di concentrazione plasmatica, compreso tra 18 e $40 \mathrm{ng} / \mathrm{ml}$, entro 1 - 4 ore dall'ingestione $[2,8]$. Oxatomide ha un elevato legame con le proteine plasmatiche (91\%) e un'emivita di distribuzione di circa 4 ore; i tessuti in cui si raggiungono le concentrazioni maggiori sono fegato, pancreas, polmoni e, in misura minore, reni e cuore. Il metabolismo è epatico e consiste principalmente di due vie metaboliche: l'idrossilazione aromatica, che porta alla formazione del principale metabolita eliminato con le feci (circa $20-30 \%$ della dose somministrata), e la N-dealchilazione, con formazione del principale metabolita eliminato con le urine, che costituisce circa il $13 \%$ della dose [2]. Una percentuale trascurabile del farmaco è eliminato in forma immodificata con le urine. Dato il metabolismo prevalentemente epatico, in caso di insufficienza renale non sono necessari aggiustamenti posologici. L'emivita plasmatica di oxatomide è compresa tra le 14 e le 30 ore [9], con una durata d' azione sufficientemente lunga da permetterne la somministrazione in una o due dosi giornaliere.

Sono stati condotti studi di farmacocinetica anche in bambini e in neonati pre-termine $[10,11]$, con risultati sostanzialmente sovrapponibili a quelli ottenuti negli adulti, dato importante vista la rilevanza della patologia allergica nella popolazione pediatrica.

L'assorbimento percutaneo di oxatomide è piuttosto scarso, con concentrazioni plasmatiche che non superano i $5 \mathrm{ng} / \mathrm{ml}$ dopo una settimana di applicazioni quotidiane di un gel al 5\% [12], per cui l'uso topico risulta virtualmente privo di effetti sistemici.

\section{Farmacologia clinica}

Per definire con precisione 1'attività antiallergica e il potenziale di impiego clinico di oxatomide sull'uomo sono stati condotti numerosi studi mediante test di provocazione, sia specifici che aspecifici. L'effetto inibitorio di oxatomide sulle reazioni da ipersensibilità è stato studiato a livello bronchiale, congiuntivale, nasale e cutaneo.

I risultati dei test di provocazione condotti per la valutazione dell'effetto protettivo di oxatomide sul broncospasmo indotto da esercizio, da metacolina o allergeni specifici sono stati analizzati da Oggiano e Nicora nel 1997 [13]. In totale, il farmaco è stato valutato su 161 soggetti, di cui 105 bambini al di sotto dei 13 anni. In generale, il farmaco è apparso in grado di esercitare un discreto effetto protettivo, non sempre significativo da un punto di vista statistico per le limitate dimensioni dei campioni esaminati. Nel più ampio di questi studi, condotto in aperto su 60 bambini atopici, dopo 3 settimane di trattamento con $1 \mathrm{mg} / \mathrm{kg} /$ die di oxatomide ben il $60 \%$ dei pazienti è risultato protetto da provocazione aspecifica con metacolina. Al controllo successivo, effettuato dopo altre 3 settimane di terapia, l'effetto protettivo è stato osservato in un ulteriore $30 \%$ del campione. 
Ciprandi et al. [14] hanno indagato l'effetto protettivo di oxatomide a livello congiuntivale, dimostrando che dopo una settimana di trattamento con $60 \mathrm{mg} / \mathrm{die}$ di oxatomide, la reazione congiuntivale alla provocazione con allergene (Parietaria judaica) è significativamente ridotta, sia come valutazione dell'intensità della sintomatologia (iperemia, lacrimazione, prurito-bruciore), sia come misurazione delle cellule infiammatorie e dell'espressione delle molecole di adesione, con inibizione della risposta immediata e di quella ritardata.

La capacità di oxatomide di inibire la risposta cutanea e nasale a istamina e allergeni specifici è stata saggiata in due studi $[15,16]$. I risultati indicano che oxatomide esercita un effetto protettivo statisticamente superiore a placebo, sia negli adulti che nei bambini, evidenziato come riduzione della dimensione dei pomfi e della reattività nasale dopo provocazione. Anche l'applicazione topica di oxatomide è risultata in grado di ridurre le dimensioni del pomfo da istamina e da allergene specifico nello studio di Sacerdoti e coll. [17].

\section{EFFICACIACLINICA}

L'efficacia di oxatomide come farmaco antiallergico è stata studiata nelle diverse patologie da ipersensibilità. Per quanto riguar- da le manifestazioni respiratorie, sono stati condotti studi clinici su pazienti affetti da' asma bronchiale e da rinite allergica, sia perenne che stagionale. In ambito dermatologico, le ricerche effettuate hanno riguardato varie patologie, tra cui dermatite atopica, orticaria acuta e cronica e altre dermopatie pruriginose. Sono state condotte ricerche d'efficacia e tollerabilità anche nella popolazione pediatrica, dato molto importante, vista l'elevata frequenza con cui le patologie allergiche si manifestano in questa fascia d'età.

\section{Allergie respiratorie}

Nel corso degli ultimi decenni due concetti hanno favorito un corretto approccio terapeutico al paziente con allergia respiratoria. In primo luogo, si è riconosciuta la continuità morfo-funzionale dell' apparato respiratorio, e conseguentemente la connessione esistente fra rinite ed asma. L'allergia respiratoria è dunque una patologia che interessa l'intero apparato respiratorio e può manifestarsi clinicamente come rinite, asma od entrambi, in successione o in associazione [18]. E' infatti noto che la rinite è spesso associata a iperreattività bronchiale e costituisce un fattore di rischio per lo sviluppo di asma e che rinite ed asma condividono lo stesso meccanismo patogenetico $[19,20]$. Da un punto di vista terapeutico ciò significa che l'adeguato tratta-

\begin{tabular}{|c|c|c|c|c|c|}
\hline $\begin{array}{l}\text { Rif. } \\
\text { Bibl. }\end{array}$ & Pazienti & Disegno studio & Dose O (mg/die) & $\begin{array}{c}\text { Durata } \\
\text { (gg) }\end{array}$ & Risultati con oxatomide \\
\hline 24 & $32 \mathrm{~b}$ & A & $1 / \mathrm{kg}$ di peso o 60 & 30 & $\begin{array}{l}\text { Miglioramento significativo } \\
\text { dei sintomi, con eccezione } \\
\text { dell'ostruzione nasale }\end{array}$ \\
\hline 22 & $30 \mathrm{a}$ & DC vs placebo & 60 & 30 & $\begin{array}{l}\text { Miglioramento significativo } \\
\text { dei sintomi nel } 77 \% \text { dei } \mathrm{pz} \\
\mathrm{O}>\text { placebo }(\mathrm{p}<0,05)\end{array}$ \\
\hline 25 & $20 \mathrm{a}$ & $\mathrm{C}$ vs $\mathrm{T}$ & 60 & 30 & $\begin{array}{l}\text { Miglioramento significativo } \\
\text { dei sintomi, } \mathrm{O}=\mathrm{T}\end{array}$ \\
\hline 23 & $28 \mathrm{~b}$ & uid vs bid & $1 / \mathrm{kg}$ di peso & 30 & $\begin{array}{l}\text { Miglioramento significativo } \\
\text { dei sintomi, uid = bid }\end{array}$ \\
\hline 26 & $20 \mathrm{a}$ & DC-CO vs T & 60 & 14 & $\begin{array}{l}\text { Miglioramento significativo } \\
\text { dei sintomi, } \mathrm{O}=\mathrm{T}\end{array}$ \\
\hline 27 & $90 \mathrm{a}$ & DC vs D & $1-2 / \mathrm{kg}$ di peso & 120 & $\begin{array}{l}\text { Miglioramento significativo } \\
\text { dei sintomi, } O>D\end{array}$ \\
\hline 28 & $56 \mathrm{a}$ & DC vs placebo & 60 & 30 & $\begin{array}{l}\text { Miglioramento significativo } \\
\text { dei sintomi, } \mathrm{O}>\text { placebo }\end{array}$ \\
\hline 29 & $63 \mathrm{a}$ & DC vs placebo & 60 & Pollinazione & $\begin{array}{l}\text { Miglioramento significativo } \\
\text { dei sintomi, } O>\text { placebo }\end{array}$ \\
\hline
\end{tabular}

Tabella 1

Studi clinicicon oxatomide: trattamento delle rinocongiutiviti

Legenda:

a adulti, b bambini, A aperto, $\mathrm{C}$ controllato, $\mathrm{DC}$ doppio cieco, $\mathrm{CO}$ cross-over, $\mathrm{O}$ oxatomide, $\mathrm{CE}$ cetirizina, T terfenadina, D difenidramina, CL clorfenamina, uid 1 somministrazione al giorno, bid due somministrazioni al giorno 
mento della rinite può influenzare positivamente anche l'asma.

Il secondo concetto chiave è quello già esposto della flogosi minima persistente, cioè di un processo infiammatorio che perdura anche quando la carica allergenica è modesta ed il paziente è oligosintomatico [21]. La flogosi a livello tessutale, innescata e mantenuta dal sistema immunitario, è responsabile dello sviluppo di danni anatomo-funzionali persistenti ed irreversibili. Da un punto di vista terapeutico ciò significa che la terapia deve controllare anche la flogosi minima persistente e ciò si ottiene grazie a farmaci antiallergici del tipo "dual action", somministrati in modo continuativo per tutta la durata dell'esposizione allergenica, anche quando questa è modesta ed incapace di causare i sintomi tipici.

L'efficacia di oxatomide nel trattamento della rinite allergica, sia stagionale che perenne, è stata documentata in numerosi studi clinici (Tabella 1)

Oxatomide, alla dose di $60 \mathrm{mg} / \mathrm{die}$ in somministrazione unica giornaliera, è stato confrontato con placebo in uno studio randomizzato in doppio cieco su 30 soggetti affetti da rino-congiuntivite stagionale [22]. Dopo 4 settimane di trattamento, il miglioramento sintomatologico è risultato evidente sia rispetto al gruppo di controllo che rispetto al placebo, con risultati soddisfacenti in 10 soggetti su 13 inclusi nel gruppo sottoposto a trattamento farmacologico attivo.

Un altro studio ha confrontato l'efficacia di due schemi posologici di oxatomide in bambini affetti da rino-congiuntivite allergica perenne [23]. I ventotto bambini arruolati, di età compresa tra 1 e 12 anni, sono stati suddivisi in due gruppi di trattamento, di cui il primo ha ricevuto per un mese oxatomide alla dose di $1 \mathrm{mg} / \mathrm{kg} /$ die in un'unica somministrazione, mentre ai soggetti del secondo gruppo è stata somministrata la medesima dose, ma frazionata in due somministrazioni giornaliere. Al termine dei 30 giorni di trattamento, il quadro clinico e rinoscopico dei bambini allergici è risultato decisamente migliorato, in maniera equivalente nei due gruppi, con differenze tra valori basali e finali che sono risultate statisticamente significative per quanto riguarda l'ostruzione nasale, la rinorrea, gli starnuti e il prurito nasale; il numero di eosinofili nel secreto nasale, inoltre, si è ridotta di circa il 50\% in entrambi i gruppi. I risultati dello studio confermano l'efficacia di oxatomide nella terapia della rino-congiuntivite allergica e indicano che i due schemi posologici esaminati producono risultati confrontabili.

In uno studio analogo [24] sono stati valutati soggetti erano di età compresa tra i 5 e i 18 anni, affetti da forme stagionali e perenni di rinocongiuntivite allergica. Anche questo lavoro sperimentale ha confermato l'equivalenza terapeutica delle due modalità di somministrazione, che hanno prodotto un miglioramento dell'intensità di starnuti, prurito nasale e occhiaie, mentre è lievemente peggiorata l'ostruzione nasale. $\mathrm{Al}$ termine del trattamento, i pazienti erano esenti da rinorrea, lacrimazione, prurito e iperemia congiuntivale.

L'efficacia di oxatomide nella gestione della sintomatologia rinitica è stata anche confrontata con altri farmaci antistaminici di uso consolidato, quali terfenadina e cetirizina.

Oxatomide è stato confrontato con terfenadina in due studi controllati condotti in doppio cieco. Nel primo sono stati arruolati 20 adulti affetti da rinite perenne, con allergia documentata agli antigeni degli acari. Il primo gruppo ha ricevuto oxatomide, alla dose di $60 \mathrm{mg} / \mathrm{die}$ in unica somministrazione giornaliera, mentre il secondo terfenadina $120 \mathrm{mg} / \mathrm{die}$ in due somministrazioni giornaliere. I risultati hanno dimostrato la pari efficacia e tollerabilità dei trattamenti farmacologici, con riduzione della gravità dei sintomi oscillante tra il 70\% e il 98\%. [25]

Il secondo degli studi di confronto tra oxatomide e terfenadina è stato condotto su soggetti affetti da allergia professionale, trattandosi di panettieri e pasticcieri allergici alla farina di frumento. Questo studio è stato condotto con un disegno in doppio cieco a crossover; i due trattamenti, della durata di 14 giorni e separati da una settimana di wash-out, consistevano nella somministrazione di oxatomide $60 \mathrm{mg} / \mathrm{die}$ in due somministrazioni, o di terfenadina $120 \mathrm{mg} / \mathrm{die}$, sempre in due somministrazioni giornaliere. Al termine dello studio è stato evidenziato un significativo miglioramento della gravità della sintomatologia con entrambi i trattamenti. I test di provocazione nasale specifica hanno comunque messo in evidenza un maggiore effetto protettivo di oxatomide. Inoltre, al termine dello studio, la maggioranza dei pazienti $(75 \%)$ ha preferito continuare il trattamento con oxatomide, piuttosto che con terfenadina. [26]

In uno studio condotto da Van der Bijl et al [27] su 90 pazienti, oxatomide $(1-2 \mathrm{mg} / \mathrm{kg} / \mathrm{die})$ è risultato superiore a difenidramina $(0,8-1,6 \mathrm{mg} /$ die) nel ridurre la sintomatologia della rinite stagionale.

Nel complesso, il corpo delle evidenze scientifiche indica che oxatomide è un farmaco efficace nella terapia delle rino-congiuntiviti allergiche, sia stagionali che perenni, alla dose di $60-120 \mathrm{mg} / \mathrm{die}$ negli adulti e di $1-2 \mathrm{mg} / \mathrm{kg} / \mathrm{die}$ nei bambini, e risulta efficace almeno quanto altri antistaminici di uso consolidato.

Per quanto riguarda gli studi clinici condotti utilizzando oxatomide per il trattamento sintomatico dell' asma bronchiale, la review di 
Oggiano e Nicora ha individuato un totale di 964 pazienti (Tab. 2), di età compresa tra gli 8 mesi e i 90 anni. Le dosi più comunemente utilizzate in questi studi sono state $60 \mathrm{mg} / \mathrm{die}$, in una o due somministrazioni, negli adulti e 1 $\mathrm{mg} / \mathrm{kg} /$ die nei pazienti pediatrici [13].

L'efficacia di oxatomide è risultata migliore nei casi di asma allergico di intensità lievemoderata. Dall'analisi dei risultati di questi studi appare che l'efficacia di oxatomide sia tendenzialmente migliore nei bambini e negli adolescenti.

Negli studi controllati, che hanno confrontato oxatomide con placebo o con ketotifene, l'efficacia del farmaco è sempre risultata superiore al placebo e al farmaco di confronto. Quando confrontato con sodio cromoglicato, oxatomide ha mostrato un profilo di efficacia molto simile, per quanto sia risultato in grado di esercitare un buon effetto terapeutico in pazienti che in precedenza non avevano risposto a tale farmaco.

Diversi studi hanno valutato l'associazione di oxatomide e salbutamolo: i risultati evidenziano la capacità dell'associazione di indurre un risparmio di farmaci antiasmatici, in particolare di cortisonici topici e di broncodilatatori [30,31].

\begin{tabular}{|c|c|c|c|c|c|}
\hline Pazienti & $\begin{array}{l}\text { Disegno } \\
\text { studio }\end{array}$ & Controllo & $\begin{array}{l}\text { Dose O } \\
\text { (mg/die) }\end{array}$ & $\begin{array}{c}\text { Durata } \\
\text { (sett.) }\end{array}$ & Risultati con oxatomide \\
\hline $15 \mathrm{a}$ & $\mathrm{DC}-\mathrm{CO}$ & Placebo & 90 & 4 & $\uparrow \mathrm{PEF}$ \\
\hline $40 \mathrm{a}$ & A & ---- & $60-120+S$ & $2-3$ & $\begin{array}{l}\text { Miglioramento significativo di sintomi } \\
\text { e PEF; riduzione consumo altri } \\
\text { antiasmatici }\end{array}$ \\
\hline $126 \mathrm{a}$ & A & ---- & $60-120$ & 6 & $\begin{array}{l}\text { Miglioramento lieve-moderato in } \\
\text { pazienti non responsivi a DSCG }\end{array}$ \\
\hline $10 \mathrm{a}$ & A & ---- & $30-60$ & $22-77$ & Efficace nel $70 \%$ dei pazienti \\
\hline $40 \mathrm{a}$ & $\mathrm{C}$ & $\mathrm{O}+\mathrm{S}$ & $60-90$ & 3 & $\begin{array}{l}\text { L'associazione determina } \\
\text { miglioramento funzione respiratoria }\end{array}$ \\
\hline $67 \mathrm{~b}$ & A & ---- & $1 / \mathrm{kg}$ di peso & 4 & $\begin{array}{l}\text { Miglioramento nel } 80 \% \text {, buono o } \\
\text { marcato nel } 56 \% \text { dei pazienti }\end{array}$ \\
\hline $286 \mathrm{a}$ & DC & $\begin{array}{l}\text { DSCG } \\
\text { placebo }\end{array}$ & 60 & 6 & $\begin{array}{l}\text { Miglioramento nel } 60 \% \text { dei pazienti } \\
\text { trattati con O, nel } 68 \% \text { con DSCG e } \\
\text { nel } 47 \% \text { con placebo }\end{array}$ \\
\hline $14 \mathrm{~b}$ & A & ---- & $\begin{array}{c}0,76-1,95 / \mathrm{kg} \\
\text { di peso }\end{array}$ & 18 & $\begin{array}{l}\text { Miglioramento medio-buono nel } 64 \% \\
\text { e medio-lieve nel } 79 \% \text { dei pazienti }\end{array}$ \\
\hline $40 \mathrm{~b}$ & A & ---- & $20-60+S$ & 3 & Risultati buoni nell' $80 \%$ dei casi \\
\hline $40 \mathrm{a}$ & $\mathrm{C}$ & $\mathrm{O}+\mathrm{S}$ & $30-120$ & 2 & $\mathrm{O}+\mathrm{S}$ più efficace di $\mathrm{O}$ \\
\hline $60 \mathrm{~b}$ & $\mathrm{C}$ & $\mathrm{K}$ & $25-50$ & 12 & $\begin{array}{l}\text { Controllo sintomatico migliore e più } \\
\text { rapido con } \mathrm{O}\end{array}$ \\
\hline $40 \mathrm{a}$ & $\mathrm{C}$ & $\mathrm{O}+\mathrm{S}$ & 60 & $12-22$ & $\begin{array}{l}\text { O efficace nel } 55 \% \text { dei } \mathrm{pz}, \mathrm{O}+\mathrm{S} \text { nel } \\
90 \%\end{array}$ \\
\hline $20 \mathrm{a}$ & $\mathrm{C}$ & O 30 bid & 60 uid & 8 & Schema uid superiore a schema bid \\
\hline $40 \mathrm{a}$ & $\mathrm{C}$ & S & $60+S$ & 10 & $\begin{array}{l}\mathrm{O}+\mathrm{S} \text { efficace nel } 95 \% \text { dei } \mathrm{pz}, \mathrm{S} \\
\text { nell' } 80 \%\end{array}$ \\
\hline $30 \mathrm{a}$ & $\mathrm{C}$ & $\mathrm{K}$ & 60 & 12 & $\begin{array}{l}\text { O efficace nel } 70 \% \text { dei pz, K nel } \\
40 \%\end{array}$ \\
\hline $40 \mathrm{~b}$ & $\mathrm{C}$ & $S$ & $60+S$ & $10-21$ & $\begin{array}{l}\mathrm{O}+\mathrm{S} \text { : efficacia ottima nel } 95 \% \text {, } \\
\text { tollerabilità ottima nel } 100 \%\end{array}$ \\
\hline $16 \mathrm{~b}$ & DC & Placebo & 1/ kg di peso & 8 & Controllo completo dei sintomi con $\mathrm{O}$ \\
\hline
\end{tabular}

Tabella 2

Studi clinici con oxatomide: trattamento dell'asma

Legenda:

a adulti, b bambini, A aperto, $\mathrm{C}$ controllato, $\mathrm{DC}$ doppio cieco, $\mathrm{CO}$ cross-over, $\mathrm{O}$ oxatomide, $\mathrm{S}$ salbutamolo, K ketotifene, DSCG cromoglicato disodico, PEF picco di flusso espiratorio, uid 1 somministrazione al giorno, bid due somministrazioni al giorno 
Oxatomide non è propriamente un farmaco antiasmatico, come messo anche in evidenza da una recente review pubblicata da esperti del Cochrane Database [32]. Il ruolo dell'oxatomide nell' asma è piuttosto preventivo, nell'ambito di una terapia di fondo, e "coadiuvante", in considerazione del forte legame tra alte e basse vie respiratorie. La review di Oggiano e Nicora[13] dimostra che il farmaco, grazie alla sua duttilità e maneggevolezza può risultare utile nei pazienti asmatici. Infatti la duplice azione antiallergica, che unisce l'attività antistaminica a quella di inibizione dell'infiammazione allergica, coniugata all'ottima tollerabilità, rende oxatomide un candidato molto interessante per la gestione di questo tipo di pazienti. Oltre a migliorare il controllo della sintomatologia respiratoria, oxatomide può ridurre il consumo degli altri farmaci antiasmatici e abolire la necessità di trattamenti specifici per le altre manifestazioni allergiche, semplificando notevolmente lo schema terapeutico.

Più in generale, oxatomide, grazie alle sue caratteristiche farmacologiche, può essere considerato un farmaco di prima linea nel paziente affetto da allergia respiratoria, in cui è in grado di prevenire la progressione da rinite ad asma e controllare i sintomi delle alte e delle basse vie respiratorie, che si trovano spesso abbinati.

\section{Manifestazioni cutanee}

Oxatomide è stato valutato nella terapia di molteplici patologie di interesse dermatologico e allergologico, dall'orticaria cronica alla dermatite atopica; in questo ambito è stato utilizzato sia per via sistemica che per via topica.

\section{Formulazione orale}

Gli studi clinici condotti per la valutazione di oxatomide nel trattamento delle dermopatie sono stati passati in rassegna in un lavoro di Stingeni et al. del 1998 [33]. Gli autori di questa review hanno individuato circa 30 studi condotti con l'utilizzo di oxatomide per via orale (Tab. 3) in un totale di oltre 1970 dermopazienti, di età compresa tra 6 mesi e 90 anni.

Le patologie più studiate in questo ambito sono l'orticaria cronica negli adulti e la dermatite atopica nei bambini.

Per quanto riguarda l'orticaria cronica, il trattamento con oxatomide, solitamente alla dose di $60 \mathrm{mg} /$ die e talvolta a dosaggio doppio, è generalmente risultato in grado di indurre la risoluzione o un significativo miglioramento dei sintomi nel $65-85 \%$ dei pazienti trattati. L'azione del farmaco è sempre risultata superiore al placebo e, quando confrontata con altri farmaci, è risultata almeno altrettanto effi- cace. In particolare, negli studi di confronto con altri farmaci dalle indicazioni simili, oxatomide è risultato costantemente superiore a ketotifene, mequitazina e terfenadina e paragonabile a clemastina e clorfeniramina. La spiccata attività di oxatomide nell'orticaria cronica è da imputarsi alla sua caratteristica doppia azione antiallergica: se infatti l'attività antistaminica produce un rapido sollievo della sintomatologia, l'azione sui mediatori cellulari dell'infiammazione è in grado di modificare l'evoluzione temporale della dermopatia, riducendo la flogosi sottostante e consentendo la riduzione delle terapie topiche spesso necessarie al controllo dell'eritema e dei pomfi. Oxatomide è in grado di controllare efficacemente il prurito, anche quando si manifesta in condizioni non mediate dal sistema immunitario. L'attività antipruriginosa è di notevole importanza, in quanto al sollievo del paziente si aggiunge la prevenzione o la remissione delle lesioni secondarie a grattamento, quali escoriazione e lichenificazione.

Il trattamento della dermatite atopica con oxatomide è stato valutato in numerosi studi clinici controllati. Il trattamento, alla dose di 1 $\mathrm{mg} / \mathrm{kg} / \mathrm{die}$, talvolta alla dose doppia, è risultato in grado di determinare un miglioramento della sintomatologia nella maggior parte dei pazienti, in genere dopo 15-30 giorni di terapia. È da sottolineare che gli studi sono stati condotti anche su casistiche particolari, comprendenti anche lattanti, per cui i buoni risultati ottenuti, associati alla buona tolerabilità, depongono per un rapporto rischio/beneficio molto favorevole. Ciò è importante anche per la natura della dermatite atopica, patologia in sé benigna e a risoluzione spontanea, ma dal decorso cronico-recidivante e dalla sintomatologia spesso intensa. La disponibilità di un farmaco come oxatomide, efficace e ben tollerato anche per periodi prolungati di tempo, ha quindi la potenzialità di migliorare profondamente la qualità di vita del bambino e dei suoi familiari.

Due studi condotti su particolari gruppi di pazienti, il primo composto da bambini talassemici con ipersensibilità alla deferossamina e l'altro da pazienti in trattamento dialitico, mettono in luce l'elevata efficacia antipruriginosa e sicurezza terapeutica di oxatomide orale nella terapia delle dermopatie pruriginose.

Nel primo di questi studi [34], venti pazienti talassemici di giovane età (5-16 anni) che presentavano sintomi cutanei di ipersensibilità alla deferossamina sono stati trattati per 2 mesi con oxatomide per os, alla dose di $1 \mathrm{mg} / \mathrm{kg} / \mathrm{die}$ in due prese giornaliere. Il trattamento ha indotto un marcato miglioramento della sintomatologia (prurito, eritema, pomfi, infiltrato 
e dolore localizzati nella sede di inoculo della deferossamina) in tutti i pazienti, con risoluzione completa nel 80\% del campione.

Manescalchi e coll. [35] hanno invece dimostrato l'utilità dell'oxatomide in una particolare forma di prurito, quello da uremia cronica, che non si ritiene essere mediato dal sistema immunologico. In questo studio, dosi giornaliere di oxatomide oscillanti tra i 15 e i $60 \mathrm{mg}$ sono risultate efficaci nel ridurre l'intensità del prurito del 70-93\% dopo un mese di terapia. $\mathrm{E}$ anche interessante notare che alcuni dei pazienti arruolati in questo studio clinico non avevano precedentemente risposto ad altri antistaminici e che la sospensione del tratta- mento ha portato alla ricomparsa del sintomo, risolto però agevolmente con la ripresa della terapia.

Un'ulteriore conferma dell'efficacia antipruriginosa di oxatomide viene da uno studio di Dupont del 1984 [36] condotto su 35 pazienti anziani (età > 65 anni) affetti da prurito senile, una condizione secondaria alla degenerazione atrofica della cute dovuta all'età, spesso di intensità tale da interferire con il sonno e capace di causare disturbi psicologici. La somministrazione di oxatomide $30 \mathrm{mg}$ BID è risultata in grado di sopprimere completamente o di ridurre in maniera marcata il prurito nel $79 \%$ dei pazienti, rispetto al $31 \%$ dei pazienti

\begin{tabular}{|c|c|c|c|c|c|c|}
\hline \multirow{2}{*}{$\begin{array}{c}\text { Pazienti e } \\
\text { diagnosi }\end{array}$} & \multirow{2}{*}{$\begin{array}{c}\text { Disegno } \\
\text { studio }\end{array}$} & \multirow{2}{*}{ Controllo } & \multirow{2}{*}{$\begin{array}{c}\text { Dose O } \\
\text { (mg/die) }\end{array}$} & \multirow{2}{*}{$\begin{array}{c}\text { Durata } \\
\text { (sett.) }\end{array}$} & \multicolumn{2}{|c|}{ Risultati con oxatomide } \\
\hline & & & & & Efficacia & Tollerabilità \\
\hline $\begin{array}{c}23 \mathrm{a} \\
\text { DA, DAC, } \\
\text { ORT ac, AE }\end{array}$ & A & ---- & $30-360$ & $2-64$ & $\begin{array}{l}\text { Soddisfacente/ott- } \\
\text { ima nel } 82,6 \% \text { dei } \\
\text { pazienti }\end{array}$ & $\begin{array}{l}3 \text { casi di } \\
\text { sonnolenza } 1 \\
\text { aumento di peso }\end{array}$ \\
\hline $\begin{array}{c}32 \mathrm{a} \\
\text { ORT ac e cr }\end{array}$ & $\mathrm{CO}$ & Placebo & $120-180$ & $6-8$ & $\begin{array}{l}\text { O: miglioramento } \\
\text { in oltre il } 75 \%, \mathrm{P} \text { : } \\
\text { peggioramento }\end{array}$ & $\begin{array}{l}4 \text { casi di } \\
\text { sonnolenza } 1 \\
\text { aumento di peso }\end{array}$ \\
\hline $\begin{array}{c}83 \mathrm{a} \\
\text { ORT cr }\end{array}$ & A & ---- & $84-120$ & $12-62$ & Ottima $(70 \%)$ & $\begin{array}{l}19 \text { pazienti con } \\
\text { effetti indesiderati, } \\
5 \text { sospendono il } \\
\text { trattamento }\end{array}$ \\
\hline $\begin{array}{c}35 \mathrm{a} \\
\text { ORT cr }\end{array}$ & $\mathrm{DC}$ & Placebo & 60 & 5 & $\begin{array}{l}\text { O: buona/ottima } \\
\text { nel } 72 \% \\
(\mathrm{p}<0,001) \\
\text { Placebo: } \\
\text { buona/ottima nel } \\
23 \% \text { dei casi }\end{array}$ & $\begin{array}{l}\text { Sonnolenza in } 1 \\
\text { paziente trattato } \\
\text { con } \mathrm{O} \text { e in } 1 \\
\text { trattato con } \\
\text { placebo }\end{array}$ \\
\hline $\begin{array}{c}41 \mathrm{a} \\
\text { ORT ac }\end{array}$ & DC & Placebo & 60 & 8 & $\begin{array}{l}\text { O: buona/ottima } \\
\text { nel } 71 \% \\
(\mathrm{p}<0,001) \\
\text { Placebo: } \\
\text { buona/ottima nel } \\
\text { 14,3\% dei casi }\end{array}$ & \\
\hline $\begin{array}{c}26 \mathrm{a} \\
\text { ORT cr }\end{array}$ & $\mathrm{DC}$ & M & 60 & 5 & $\mathrm{O}>\mathrm{M}(\mathrm{p}<0,02)$ & $\begin{array}{l}\text { O e M } 3 \text { casi di } \\
\text { sonnolenza }\end{array}$ \\
\hline $\begin{array}{l}1150 \text { (a e b) } \\
\text { ORT ac, } \\
\text { DA,DAC }\end{array}$ & A & ---- & $15-60$ & 4 & $\begin{array}{l}\text { Buona/ottima } \\
\text { nel } 73 \%\end{array}$ & $\begin{array}{l}\text { EC nel } 22,3 \% \text {, } \\
\text { con sospensione } \\
\text { di O nel } 4,7 \%\end{array}$ \\
\hline $\begin{array}{l}76 \mathrm{a} \mathrm{e} \mathrm{b} \\
\text { ORT cr }\end{array}$ & A & ---- & $30-60$ & 4 & Buona nel $83 \%$ & ---- \\
\hline
\end{tabular}

Tabella 3

Studi clinici con oxatomide: trattamento delle dermopatie Modificata da Stingeni et al, 1998 [33]

\section{Legenda:}

a adulti, b bambini, A aperto, $\mathrm{C}$ controllato, DC doppio cieco, $\mathrm{CO}$ cross-over, $\mathrm{O}$ oxatomide,

M mequitazina DA dermatite atopica DAC dermatite allergica da contatto, ORT orticaria, ac acuta, cr cronica, AE angioedema, EC effetti collaterali

Nota: Solo alcuni degli studi revisionati da Stingeni et al. sono stati inclusi in tabella 
del gruppo di controllo, trattati con placebo. L'efficacia di oxatomide è risultata evidente nel ridurre l'intensità e la durata del prurito, come dimostrato anche dal fatto che i pazienti del gruppo di controllo hanno dovuto ricorrere maggiormente a medicazioni topiche per ottenere sollievo.

\section{Formulazione topica}

La valutazione dell'oxatomide per via topica (gel al 5\%) è stata condotta prevalentemente su pazienti affetti da dermopatie pruriginose localizzate, di diversa patogenesi.

Il trattamento topico è risultato in grado di controllare adeguatamente il prurito nella maggioranza dei pazienti, con regressione delle lesioni secondarie quali escoriazioni e lichenificazione, dopo 10-30 giorni di applicazioni del gel [33].

Gli studi che hanno confrontato il farmaco con un placebo hanno costantemente dimostrato la superiorità di oxatomide e anche lo studio di Locci e Del Giacco [37], che ha confrontato il gel di oxatomide con una preparazione analoga a base di desclorfeniramina in pazienti affetti da orticaria cronica, ha riscontrato una maggior efficacia di oxatomide per quanto riguarda il prurito e il numero e la dimensione dei pomfi, mentre il gel di desclorfeniramina è risultato più efficace nel ridurre l'intensità dell'eritema.

L'efficacia di oxatomide gel nel trattamento del prurito vulvare, causato da lichen sclerosus e da altre patologie genitali, è stata valutata in due studi di Origoni et al [38,39]. In entrambi gli studi, oxatomide è risultato superiore al placebo nel controllare il prurito, mentre non sono state registrate differenze significative per quanto riguarda i sintomi associati, ossia bruciore, disuria e dispareunia.

\section{Intolleranze e allergie alimentari}

In uno studio in doppio cieco condotto su 32 bambini di età compresa tra i 6 e i 35 mesi, Capristo e coll. [40] hanno valutato la capacità di oxatomide di controllare i sintomi dovuti a allergia o intolleranza alimentare. Dopo essere stati trattati per un mese con una terapia basata sull'esclusione dietetica dei prodotti a cui erano allergici o intolleranti, i pazienti hanno ricevuto oxatomide $(1 \mathrm{mg} / \mathrm{kg} / \mathrm{die}$, in due prese giornaliere) o placebo per 42 giorni, mantenendo la terapia dietetica. In entrambi i gruppi il miglioramento è risultato evidente già nel periodo di trattamento dietetico, con riduzione della sintomatologia cutanea, respiratoria e gastrointestinale, ma il gruppo trattato con oxatomide ha poi mostrato un miglioramento più marcato, in particolare per quanto riguarda i sintomi cutanei e rinitici, che avevano scarsamente risposto alla terapia di esclusione alimentare.

L'utilità di oxatomide nel controllare i sintomi delle allergie e delle intolleranze è stata anche dimostrata da Pacor et al. [41], in uno studio di confronto vs. sodio cromoglicato. Lo studio, condotto in doppio cieco con disegno a cross-over, ha coinvolto 20 soggetti affetti da orticaria o eczema di origine alimentare, con diagnosi di allergia alimentare nella metà dei casi e di intolleranza alimentare nei restanti dieci pazienti. I soggetti hanno ricevuto per 6 settimane oxatomide $60 \mathrm{mg} /$ die o sodio cromoglicato $2 \mathrm{~g} / \mathrm{die}$, seguite da tre settimane di wash-out prima di assumere il trattamento di confronto, con sequenza determinata in maniera randomizzata e in doppio cieco. Entrambi i farmaci si sono dimostrati efficaci nel controllare la sintomatologia cutanea durante i periodi di trattamento, ma i risultati evidenziano una certa superiorità di oxatomide, in grado di indurre la completa scomparsa dei pomfi nel $75 \%$ dei soggetti, delle lesioni eczematose nel $64 \%$ e del prurito nel $70 \%$, rispetto a $33 \%, 50 \%$ e $50 \%$, rispettivamente, ottenuti con sodio cromoglicato.

\section{SICUREZZAETOLLERABILITÀ}

La tollerabilità di oxatomide è risultata generalmente molto buona. Gli effetti collaterali più frequentemente segnalati sono stati quelli caratteristici degli antistaminici, cioè sonnolenza e aumento di peso, normalmente transitori.

La review di Richards et al. del 1984 [2] riporta che la sonnolenza, effetto collaterale talora gradito, specie nei bambini con forte intensità del prurito, aveva interessato circa il $10 \%$ di 1107 pazienti arruolati in studi clinici, pubblicati e non, senza di norma comportare l'interruzione del trattamento. In uno studio policentrico di confronto tra due schemi posologici di oxatomide, Benvenuti e Cavalli [42] hanno raccolto i dati di tollerabilità su 920 pazienti asmatici. Il profilo di sicurezza nella casistica adulta è risultato analogo con i due schemi posologici, con un'incidenza di eventi collaterali intorno al $15 \%$. Tre pazienti hanno sospeso il trattamento, due a causa di sonnolenza di media entità e 1 paziente per crisi d'asma. Nei soggetti pediatrici, la somministrazione di oxatomide in due prese giornaliere è stata meglio tollerata della monosomministrazione, con incidenza di eventi collaterali del 
$9 \%$ e del $15 \%$, rispettivamente. L'accettabilità del trattamento è stata valutata positivamente dalla maggior parte dei pazienti, sia adulti che bambini. Lo studio di Stingeni [34] indica che la percentuale di pazienti che hanno interrotto il trattamento con oxatomide a causa della sonnolenza è stata del 3,6\%. Gli effetti sedativi possono comunque essere controllati sia mediante adeguamento della posologia alla sensibilità individuale, sia utilizzando la monosomministrazione serale [13].

Il secondo effetto collaterale di oxatomide in ordine di frequenza, l'aumento di peso, secondario ad aumento dell' appetito, ha un'incidenza del 2,5\%, secondo la review di Richards e inferiore all'1\% nello studio di farmacovigilanza di Benvenuti e Cavalli.

Tutti gli altri effetti collaterali sono stati segnalati in una percentuale inferiore all' $1 \%$ dei soggetti trattati.

La sicurezza cardiovascolare degli antistaminici, soprattutto di quelli di seconda generazione, è di particolare interesse, dati i problemi che hanno portato al ritiro dal commercio della terfenadina. I dati di farmacovigilanza di oxatomide sono molto rassicuranti da questo punto di vista: nel triennio 1994-1996 si sono registrati solo 16 casi di tossicità cardiovascolare e 18 casi di disturbo del ritmo o della frequenza cardiaca su un totale di oltre 21 milioni di pazienti trattati. In quello stesso periodo, in Italia, l'unico evento avverso cardiaco segnalato, su circa 2.900 .000 pazienti esposti, è un caso di tachicardia [43]. L'assenza di effetti collaterali potenzialmente letali è importante per qualsiasi terapia, ma diviene fondamentale quando la cura è diretta al controllo della sintomatologia di patologie relativamente benigne, che non mettono in pericolo la vita del paziente: in questa situazione, il rischio di eventi fatali o gravemente disabilitanti, per quanto rari, è inaccettabile, riducendo in maniera sostanziale il rapporto rischio/beneficio, parametro fondamentale per la scelta razionale di qualsiasi terapia.

Anche a livello epatico oxatomide è ben tollerato, con solamente qualche caso segnalato di elevazione delle transaminasi sieriche. Oxatomide inoltre non interferisce con le funzioni immunitarie, a differenza di altre molecole che ne condividono le indicazioni.

Oxatomide risulta tollerato bene anche nelle età limite, essendo stato studiato su pazienti molto giovani (lattanti e pre-termine) e su ultraottantenni.

Anche la formulazione topica risulta ben tollerata e non sono stati segnalati casi di fototossicità. Il gel provoca bruciore o irritazione locale in una bassa percentuale di pazienti, valutata intorno all' $1-2 \%$.

\section{CONSIDERAZIONI FARMACOECONOMICHE}

La valutazione dei costi di una terapia farmacologica diviene sempre più necessaria, man mano che si riducono le risorse disponibili e aumenta, sia nel mondo sanitario che pubblico, la consapevolezza della necessità di razionalizzare la spesa. Ciò è particolarmente vero per patologie a grande impatto sociale, com'è il caso delle malattie allergiche, che hanno profondi effetti sulla qualità della vita. Inoltre $\mathrm{i}$ farmaci antistaminici per via orale sono collocati in fascia $\mathrm{C}$, cioè a totale carico dell'assistito; la loro scelta è però, ovviamente, guidata dal medico, che deve dunque avere a disposizione validi strumenti che gli permettano di decidere razionalmente tra le varie opzioni disponibili. La farmacoeconomia è la scienza che si prefigge di fornire tali strumenti, attraverso l'esplicitazione, la valorizzazione e l'analisi delle implicazioni cliniche ed economiche di una o più terapie farmacologiche.

Non sono disponibili valutazioni farmacoeconomiche dell'oxatomide nella letteratura scientifica internazionale, per cui in questo lavoro, dopo una breve analisi dei costi farmacologici puri della terapia con oxatomide, confrontati con alcune delle alternative disponibili sul mercato, si cercherà di evidenziare vantaggi e limiti del prodotto attraverso la costruzione di una "matrice dei valori", mediante la quale verranno esposti gli aspetti negativi e positivi, sia dal punto di vista clinico che economico, dell'oxatomide rispetto ai farmaci di confronto, secondo il punto di vista di tutti gli attori coinvolti nel processo decisionale.

\section{Costi di acquisto}

Una confezione di 30 compresse di $30 \mathrm{mg}$ di oxatomide (Tinset $\circledR$ - Formenti) è venduta al pubblico a 8 euro. Il costo/settimana di terapia risulta, considerando una DDD di $60 \mathrm{mg}$ die, pari a 3,73 euro. Se invece consideriamo la posologia media giornaliera prescritta, rilevata dallo Studio Prescrizioni Mediche (SPM), notiamo un dosaggio pari a 1,6 unità posologiche per un totale di $48 \mathrm{mg} / \mathrm{die}$, con un costo /settimana di terapia di 2,87 euro. La Tabella 5 presenta $\mathrm{i}$ costi/settimana comparati di alcuni tra $i$ farmaci antiallergici più comunemente utilizzati nel nostro paese, ai dosaggi giornalieri definiti DDD (Defined Daily Dose) dal WHO Collaborating Centre for Drug Statistics Methodology [44] per le molecole classificate nella classe R06A (antistaminici per via sistemica) dell' ATC e al dosaggio medio prescritto PDD (Prescribed Daily Dose), secondo quanto rilevato da SPM ( Servizio Prescrizioni Mediche) a settembre del 2003 [45]. 


\begin{tabular}{|c|c|c|c|c|c|c|c|c|c|}
\hline $\begin{array}{l}\text { Farmaco e unità } \\
\text { posologica }\end{array}$ & Prezzo & $\begin{array}{l}\mathbf{N}^{\circ} \mathbf{U} \text {. } \\
\text { pos. }\end{array}$ & $\begin{array}{c}\text { Costo/U. } \\
\text { pos. }\end{array}$ & $\begin{array}{c}\text { DDD } \\
\text { [U. pos.] }\end{array}$ & $\begin{array}{c}\text { PDD } \\
\text { (U. pos.) }\end{array}$ & $\begin{array}{l}\text { Cost } \\
\text { DDD }\end{array}$ & $\begin{array}{l}\text { o/die } \\
\text { PDD }\end{array}$ & $\begin{array}{l}\text { Costc } \\
\text { DDD }\end{array}$ & $\begin{array}{l}\text { /sett. } \\
\text { PDD }\end{array}$ \\
\hline $\begin{array}{l}\text { OXATOMIDE } \\
30 \mathrm{mg}\end{array}$ & 8,00 & 30 & 0,26 & 2 & 1,6 & $\mathbf{0 , 5 2}$ & 0,41 & 3,64 & 2,87 \\
\hline $\begin{array}{l}\text { LORATADINA } \\
\text { 10mg (Costo minimo) }\end{array}$ & 11,60 & 20 & 0,58 & 1,0 & 1,0 & 0,58 & 0,58 & 4,06 & 4,06 \\
\hline $\begin{array}{l}\text { LORATADINA } \\
\text { 10mg (Costo massimo) }\end{array}$ & 12,90 & 20 & 0,65 & 1,0 & 1,0 & 0,65 & 0,65 & 4,52 & 4,52 \\
\hline $\begin{array}{l}\text { DESLORATADINA } \\
5 \mathrm{mg}\end{array}$ & 13,90 & 20 & 0,70 & 1,0 & 1,0 & 0,70 & 0,70 & 4,87 & 4,87 \\
\hline $\begin{array}{l}\text { DESLORATADINA } \\
5 \mathrm{mg}\end{array}$ & 5,60 & 7 & 0,80 & 1,0 & 1,1 & 0,80 & 0,88 & 5,60 & 6,16 \\
\hline $\begin{array}{l}\text { EBASTINA } \\
10 \mathrm{mg}\end{array}$ & 16,12 & 30 & 0,54 & 1,0 & 1,0 & 0,54 & 0,54 & 3,76 & 3,76 \\
\hline $\begin{array}{l}\text { EBASTINA } \\
20 \mathrm{mg}\end{array}$ & 17,80 & 20 & 0,89 & 1,0 & 1,0 & 0,89 & 0,89 & 6,23 & 6,23 \\
\hline $\begin{array}{l}\text { CETIRIZINA } \\
10 \mathrm{mg}\end{array}$ & 4,50 & 7 & 0,64 & 1,0 & 1,1 & 0,64 & 0,71 & 4,50 & 4,95 \\
\hline $\begin{array}{l}\text { CETIRIZINA } \\
10 \mathrm{mg}\end{array}$ & 11,50 & 20 & 0,58 & 1,0 & 1,1 & 0,58 & 0,63 & 4,03 & 4,43 \\
\hline $\begin{array}{l}\text { CLORFENAMINA } \\
4 \mathrm{mg}\end{array}$ & 8,30 & 20 & 0,42 & 3,0 & 1,9 & 1,25 & 0,79 & 8,72 & 5,52 \\
\hline $\begin{array}{l}\text { TERFENADIA } \\
60 \mathrm{mg}\end{array}$ & 5,89 & 30 & 0,20 & 2,0 & 1,0 & 0,39 & 0,20 & 2,75 & 1,37 \\
\hline $\begin{array}{l}\text { FEXOFENADINA } \\
120 \mathrm{mg}\end{array}$ & 10,90 & 20 & 0,55 & 1,0 & 1,0 & 0,55 & 0,55 & 3,82 & 3,82 \\
\hline $\begin{array}{l}\text { FEXOFENADINA } \\
180 \mathrm{mg}\end{array}$ & 11,97 & 20 & 0,60 & 1,0 & 1,1 & 0,60 & 0,66 & 4,19 & 4,61 \\
\hline $\begin{array}{l}\text { KETOTIFENE } \\
2 \mathrm{mg}\end{array}$ & 7,50 & 15 & 0,50 & 1,0 & 1,2 & 0,50 & 0,60 & 3,50 & 4,20 \\
\hline
\end{tabular}

\section{Tabella 4}

Costo farmaceutico al pubblico di oxatomide e altri antiallergici (cost $i$ e prezzi sono indicati in euro)
Tinset ${ }^{\circledR}$, indipendentemente dalla fonte utilizzata, risulta essere il farmaco più vantaggioso in termini di costo beneficio se paragonato tra quelli più utilizzati nella sua classe.

Infatti il prezzo di acquisto di oxatomide, come risulta evidente dalla tabella, è tra i più bassi nella sua categoria, considerando che solo terfenadina, farmaco efficace ma gravato da tossicità cardiovascolare e alto potenziale di interazioni farmacologiche, e ketotifene, che condivide solo in parte le indicazioni di oxatomide, essendo indicato soprattutto in profilassi, si collocano a un livello di prezzo inferiore.

\begin{tabular}{|c|c|c|}
\hline \multicolumn{3}{|c|}{ Prospettiva del paziente } \\
\hline Valore & Positivo & Negativo \\
\hline Clinico & $\begin{array}{l}\text { Riduzione dei sintomi e del rischio di } \\
\text { progressione e cronicizzazione della } \\
\text { patologia allergica } \\
\text { Buona tollerabilità in un'elevata } \\
\text { percentuale di pazienti } \\
\text { Minimo rischio di effetti collaterali gravi } \\
\text { e potenzialmente letali } \\
\text { Riduzione della necessità di altre } \\
\text { terapie; efficace sui diversi sintomi } \\
\text { allergici (respiratori e cutanei) } \\
\text { Flessibilità della posologia (UID o } \\
\text { BID) e della formulazione }\end{array}$ & $\begin{array}{l}\text { Inefficace in alcuni pazienti } \\
\text { Necessità di associare altri farmaci nei } \\
\text { casi gravi e negli asmatici } \\
\text { Possibile comparsa di sonnolenza }\end{array}$ \\
\hline Economico & $\begin{array}{l}\text { Riduzione della perdita di produttività e } \\
\text { aumento della qualità di vita del } \\
\text { paziente e del nucleo familiare }\end{array}$ & $\begin{array}{l}\text { Costo farmaceutico da coprire "di } \\
\text { tasca propria" }\end{array}$ \\
\hline
\end{tabular}

Tabella 5a

Analisi dei valori: prospettiva del paziente 


\section{Prospettiva del medico}

\begin{tabular}{lll} 
Valore & Positivo & Negativo \\
\hline Clinico & $\begin{array}{l}\text { Soddisfazione del paziente, con } \\
\text { sollievo dei sintomi } \\
\text { Azione sia preventiva che sintomatica } \\
\end{array}$ & $\begin{array}{l}\text { Insoddisfazione del paziente nei casi di } \\
\text { inefficacia o scarsa tollerabilità }\end{array}$ \\
& $\begin{array}{l}\text { Semplice prescrizione grazie alla } \\
\text { scarsezza di avvertenze e } \\
\text { controindicazioni } \\
\end{array}$ & \\
& $\begin{array}{l}\text { Non necessari controlli strumentali e di } \\
\text { laboratorio per monitorare tossicità }\end{array}$ & \\
& $\begin{array}{l}\text { Prescrizione efficiente, senza rischio } \\
\text { professionale particolare }\end{array}$ & Necessità di prescrizioni multiple nei \\
Economico & & \\
& &
\end{tabular}

\section{Prospettiva del SSN}

\begin{tabular}{lll} 
Valore & Positivo & Negativo \\
\hline Clinico & $\begin{array}{l}\text { Controllo sintomatico dei pazienti con } \\
\text { buona tollerabilità in un'elevata } \\
\text { percentuale dei casi }\end{array}$ & $\begin{array}{l}\text { Insoddisfazione del paziente nei casi di } \\
\text { inefficacia o di scarsa tollerabilità, con } \\
\text { necessità di revisione della terapia }\end{array}$ \\
Economico & $\begin{array}{l}\text { Nessuna spesa diretta per l'acquisto } \\
\text { Riduzione del fabbisogno di altri } \\
\text { farmaci rimborsati } \\
\text { Liberazione di risorse sanitarie grazie al } \\
\text { controllo sintomatico } \\
\text { Nessuna spesa per gestione tossicità }\end{array}$ & $\begin{array}{l}\text { Costi per la gestione degli eventi } \\
\text { avversi, peraltro rari. }\end{array}$ \\
&
\end{tabular}

\section{Prospettiva della società}

\begin{tabular}{lll} 
Valore & Positivo & Negativo \\
\hline Clinico & $\begin{array}{l}\text { Miglioramento della salute e della } \\
\text { qualità di vita dei cittadini }\end{array}$ & Reazioni avverse e casi di inefficacia \\
Economico & $\begin{array}{l}\text { Aumento della produttività lavorativa } \\
\text { o scolastica degli allergici e dei loro } \\
\text { familiari } \\
\text { Posti di lavoro nellindustria } \\
\text { farmaceutica }\end{array}$ & $\begin{array}{l}\text { Costo del farmaco (vedi paziente) } \\
\text { Tale costo è probabilmente } \\
\text { ampiamente compensato dai risparmi } \\
\text { indotti sul costo di malattia e pertanto } \\
\text { la società nel complesso ha un } \\
\text { beneficio economico netto }\end{array}$ \\
\hline
\end{tabular}

\section{Costo di malattia}

Il costo complessivo delle patologie allergiche, asma compreso, in Italia non è stato compiutamente stimato, ma bastano alcune cifre per evidenziare come il loro impatto, economico ma non solo, sia sostanziale. Recenti studi epidemiologici condotti per ottenere una stima della prevalenza della rinite allergica in Italia hanno fornito risultati compresi tra il $15 \mathrm{e}$ il $18 \%[46,47]$ della popolazione generale, con picchi più alti in particolari gruppi di soggetti. Il costo totale annuale di un paziente affetto da rinite allergica è stato stimato in un recen- te lavoro tedesco, condotto dal punto di vista del terzo pagante (assicurazione sanitaria): le stime ottenute variano da 1,089 euro per un bambino con rinite non complicata fino a 9,287 euro per paziente adulto con rinite e asma bronchiale [48]. Una consistente quota del valore stimato totale (40-80\%) è dovuto a costi indiretti, cioè prevalentemente alla perdita di ore e di produttività nel lavoro e nella scuola, costi che possono essere ridotti con una terapia appropriata che ottenga un buon controllo sintomatologico. Le stime economiche ottenute in paesi stranieri non possono essere automaticamente trasferite alla re-

\section{Tabella 5b}

Analisi dei valori: prospettiva del medico

\section{Tabella 5c}

Analisi dei valori: prospettiva del SSN

\section{Tabella 5d}

Analisi dei valori: prospettiva della società 
altà italiana, in quanto nei diversi ambiti i costi e le strategie sanitarie possono variare in maniera anche importante, ma ci pare che possano essere utili a delineare l'ordine di grandezza del problema. Se si considera inoltre che la rinite è solo una delle numerose patologie ad eziologia allergica e che la sua prevalenza risulta in ulteriore aumento, appare evidente che il complesso delle malattie da ipersensibilità ha costi clinici, sociali ed economici molto elevati.

\section{Analisi dei valori}

Si tratta di una tecnica di valutazione economica che consiste nell' assegnare un insieme di valori ad una determinata scelta in ambito sanitario, i cui outcomes, in questo caso costi e benefici, possono variare a seconda della prospettiva adottata. Il presupposto teorico su cui si basa tale tecnica risiede nella constatazione che un outcome che appare positivo dal punto di vista del paziente, ad esempio, possa rivestire un significato differente per gli altri attori coinvolti nella decisione, come il medico, il SSN o la società nel complesso $[49,50]$. Il fine di questa analisi è quello di fornire un quadro completo delle ricadute di un trattamento nelle diverse prospettive adottate: a tal scopo è utile costruire una matrice in cui, per ogni decisore considerato, si hanno quattro caselle, corrispondenti agli incroci tra tipo di outcome, clinico e sanitario, e segno positivo e negativo del valore.

La matrice dei valori relativa all'utilizzo dell'oxatomide in Italia, secondo le prospettive del paziente, del medico, del SSN e della società è presentata in Tabella 5 ; i giudizi espressi derivano dall'attento esame della letteratura disponibile, descritta nella prima parte del lavoro.

\section{CONCLUSIONI}

Oxatomide è un farmaco con un meccanismo di azione completo che lo rende utile nella prevenzione e nel trattamento delle manifestazioni allergiche: da un lato antagonizza l'istamina a livello recettoriale e, dall'altro, inibisce l'immunoflogosi conseguente alla liberazione e produzione dei mediatori dell'infiammazione da parte delle cellule effettrici, probabilmente comportandosi da calcio-antagonista specifico per queste popolazioni cellulari.

L'efficacia e la tollerabilità di oxatomide sono state valutate in numerosi studi clinici condotti su pazienti affetti dalle diverse patologie allergiche, dimostrando la sua efficacia e sicurezza terapeutica anche in pazienti ultraottantenni, in lattanti e in neonati pretermine. Lo spettro delle indicazioni approvate per oxatomide è molto ampio, comprendendo rinite, asma, congiuntivite, orticaria, dermatite atopica e allergie alimentari.

Negli studi di confronto, oxatomide è sempre risultato superiore a placebo e di efficacia paragonabile o superiore a quella di altri farmaci antiallergici di uso consolidato. La tollerabilità del farmaco è buona, i principali effetti collaterali sono la sonnolenza e l'aumento di peso, generalmente transitori. I dati di farmacovigilanza sono molto rassicuranti, soprattutto per quanto riguarda la tossicità cardiaca.

Dal punto di vista economico, il prezzo di acquisto di oxatomide è competitivo, essendo tra i più bassi nella sua classe, a parità di indicazioni, efficacia e sicurezza.

Nel complesso si può dire che oxatomide gode di un ottimo rapporto rischio/beneficio e costo/benefico nella prevenzione e nel trattamento delle patologie allergiche.

\section{BIBLIOGRAFIA}

1 Borgers $\mathrm{M}$ et al. Morphologic evaluation of R 35443, a new antiallergicum, in guinea pig anaphylaxis. Janssen Pharmaceutica, Preclinical Res Rep 1976; 10.

2 Richards DM et al. Oxatomide. A review of its pharmacodynamic properties and therapeutic efficacy. Drugs. 1984 Mar;27(3):210-31.

3 Marone G et al. Antiinflammatory effects of oxatomide. J Investig Allergol Clin Immunol. 1999 Jul-Aug;9(4):207-14.

4 Marone G, ed. Human Basophils and Mast Cells: Biological Aspects. Basel, Karger AG 1995.

5 Marone G ed. Human Basophils and Mast Cells: Clinical Aspects. Basel, Karger AG 1995.

6 Marone G ed. Human Eosinophils: Biological and Clinical Aspects. Basel, Karger AG 2000

7 Triggiani M et al. Inhibition of Cytosolic Phospholipase A2 in Human Neutrophils by Oxatomide Int Arch Allergy Immunol 2001;124:367-70.

8 Meuldermans W et al. Plasma levels, biotransformation and excretion of oxatomide (R 35443 ) in rats, dogs and man Xenobiotica. 1984 Jun;14(6):445-62. 
9 Davies BH, Rocchiccioli K. Oxatomide plasma levels in man during chronic dosing. Pharmatherapeutica. 1983;3(6):365-9.

10 Dani C et al. Pharmacokinetics of oxatomide in preterm infants. Drugs Exp Clin Res. 2002;28(5):207-10.

11 Cavagni $\mathrm{G}$ et al. Pharmacokinetics of two oral liquid formulations of oxatomide in children. Int J Clin Pharmacol Ther Toxicol. 1992 Aug;30(8):275-6.

12 Benvenuti $\mathrm{C}$ et al. Pharmacokinetics of oxatomide given percutaneously to healthy volunteers. Biopharm Drug Dispos. 1992 Oct;13(7):495-502.

13 Oggiano N, Nicora M. L'oxatomide nell'asma bronchiale. Rassegna dei principali dati farmacologici e clinici. Pediatria Oggi Medica e Chirurgica. 1997 17(2):35-48.

14 Ciprandi $\mathrm{G}$ et al. Oxatomide reduces inflammatory events induced by allergen-specific conjunctival challenge. Ann Allergy Asthma Immunol. 1995 Nov;75(5):446-52.

15 Piacentini GL et al. Inhibition of different dosages of oxatomide or placebo on skin prick test and nasal allergen provocation J Allergy Clin Immunol 1991 Aug;88(2):218-25

16 Canessa A, Pallestrini E. Inibizione della reattività cutanea e nasale da parte dell'oxatomide. Studio in doppio cieco versus placebo. Otorinolaringol 1993;43:57-62

17 Sacerdoti G et al. Valutazione dell'inibizione delle risposte cutanee allergene-specifiche e non da parte dell'oxatomide per uso topico. G Ital Dermatol Venereol 1990;125:LV.

18 WHO Position Paper. ARIA: Allergic Rhinitis and its Impact on Asthma. Bousquet J, Kalthaev N and van Cauwenberge P. edts. JACI 2001

19 Leynaert B et al. Perennial rhinitis: an independent risk factor for asthma in nonatopic subjects. J Alllergy Clin Immunol 104: 301-304, 1999

20 Passalacqua $\mathrm{G}$ et al. The nose-lung interaction: united airways disease. Curr Opin Allergy Clin Immunol 1: 7-13, 2001

21 Ciprandi G et al. Minimal persistent inflammation is present at mucosal level in symptomatic rhinitic patients with allergy due to mites. J Allergy Clin Immunol 96: 971-979, 1995

22 D'amato $\mathrm{G}$ et al. Studio doppio cieco oxatomide vs. placebo nelle rinocongiuntiviti allergiche stagionali Giorn. It. Allergol. Immunol. Clin. 1992;2:151-6.

23 Ragno $\mathrm{V}$ et al. Oxatomide in the treatment of allergic rhinoconjunctivitis in childern: comparison of two dosage schemes Giorn. It. Allergol. Immunol. Clin. 1998;8:617-22.

24 Pozzan M. L'oxatomide nella terapia dell'oculorinite allergica in età pediatrica. Riv Pediatr Prev Soc 1996;46:13-20

25 Rossi O et al. Terapia della rinite allergica studio di confronto: oxatomide versus terfenadina. Otorinolarigol 1993;43:249-54.

26 Cirla AM et al. Oxatomide nel trattamento della rinite allergica da farina di frumento. Studio controllato verso terfenadina. Rivista di Patologia e Clinica 1998;53(2):30-41.

27 van der Bijl WJ et al. A double-blind comparison of oxatomide (R 35443$)$ and diphenhydramine in the treatment of hay fever. Laryngoscope. 1980 Jan;90(1):145-51.

28 Vannieuwenhuyse E et al. Oxatomide in the treatment of chronic allergic rhinitis. Ann Otol Rhinol Laryngol. 1982 Mar-Apr;91(2 Pt 1):175-8

29 Wood SF, Barber JH. Oxatomide in the management of hay fever - a placebo-controlled double-blind study in general practice. Clin Allergy 1981; 11:491-7.

30 Speranza D, Puppo N. Combined bronchodilator plus antihistamine treatment of asthma. Curr Ther Res 1984;36:116571.

31 Ghiringhelli G, Schiavi M. Controlled trial to evaluate the therapeutic properties of an extempore combination of a bronchodilator (salbutamol) and an antihistamine agent (oxatomide) in the treatment of intrinsic asthma. Curr Ther Res 1984;36:1158-64.

32 Hayashi Ket al. Oxatomide for stable asthma in adults and children. Cochrane Database Syst Rev. 2003;(2):CD002179.

33 Stingeni L et al. Oxatomide in dermatologia: revisione dei principali effetti farmacodinamici e clinici. Ann It Dermatol Clin Sper 1998;52:9-21.

34 Mora P, Boccaccini R. L'oxatomide nel trattamento dei sintomi da ipersensibilità alla deferossamina in bambini talassemici. Rivista di Patologia e Clinica 1998, 53(2):45-53.

35 Manescalchi $\mathrm{F}$ et al. L'oxatomide nel trattamento del prurito del paziente uremico cronico in trattamento emodialitico. Basi Raz Ter 1989; 19:339-45.

36 Dupont $\mathrm{C}$ et al. Oxatomide in the treatment of pruritus senilis. A double-blind placebo-controlled trial. Dermatologica. 1984;169(6):348-53.

37 Locci F, Del Giacco GS Treatment of chronic idiopathic urticaria with topical preparations: controlled study of oxatomide gel versus dechlorpheniramine cream. Drugs Exp Clin Res. 1991;17(8):399-403. 
38 Origoni $\mathrm{M}$ et al. Topical oxatomide: an alternative approach for the treatment of vulvar lichen sclerosus. Int $\mathbf{J}$ Gynaecol Obstet. 1996 Dec;55(3):259-64.

39 Origoni M et al. Efficacy of topical oxatomide in women with pruritus vulvae. Drugs Exp Clin Res. 1990;16(11):591-6.

40 Capristo AF et al. Double blind study oxatomide versus placebo in the treatment of food allergy in paediatrics. Archivio di Medicina Interna 1997;49:49-61

41 Pacor ML et al. Controlled study of oxatomide vs disodium chromoglycate for treating adverse reactions to food. Drugs Exp Clin Res. 1992;18(3):119-23.

42 Benvenuti C, Cavalli E. Farmacovigilanza su oxatomide nel trattamento dell'asma bronchiale allergico. Studio policentrico di confronto tra due schemi posologici su 920 casi. Rivista di Patologia e Clinica 1995; 50(3):167-86.

43 Fossati A et al. Sicurezza cardiovascolare di oxatomide. XXII Congresso Nazionale della Società Italiana di Allergologia e Immunologia Clinica 4-8 Maggio 1998, Cagliari.

44 WHO Collaborating Centre for Drugs Statistics Methodology - The ATC/DDD index - disponibile all' indirizzo web http://www.whocc.no/atcddd/

45 IMS

46 Verlato $\mathrm{G}$ et al. Is the prevalence of adult asthma and allergic rhinitis still increasing? Results of an Italian study. $\mathrm{J}$ Allergy Clin Immunol. 2003 Jun;111(6):1232-8

47 Olivieri M et al; Italian European Community Respiratory Health Survey group. Prevalence and features of allergic rhinitis in Italy. Allergy. 2002 Jul;57(7):600-6

48 Weinmann $\mathrm{S}$ et al. The costs of atopy and asthma in children: assessment of direct costs and their determinants in a birth cohort. Pediatr Allergy Immunol. 2003 Feb;14(1):18-26.

49 Kahneman D, Twersky A. Choices, values, and frames. Am Psychologist 1984;39:341-450.

50 McGlynn EAet al. Quality-of-care research in mental health: responding to the challenge. Inquiry. 1988 Spring;25(1):157-70. 